\title{
Diffuse Venous Malformation of Perineum
}

\section{SAIF ULLAH KHAN ${ }^{1}$, MD. FARIDUL ISLAM ${ }^{1}$, DIPAL KRISHNA ADHIKARY ${ }^{2}$}

${ }^{1}$ Department of Vascular Surgery, Bangabandhu Sheikh Mujib Medical University, Dhaka. ${ }^{2}$ Department of Cardiology, Bangabandhu Sheikh Mujib Medical University (BSMMU), Dhaka.

Address for Correspondance: Dr Md Saif Ullah Khan, Assistant Professor, Department of Vascular Surgery, Bangabandhu Sheikh Mujib Medical University (BSMMU), Dhaka. Email: drsaifkhanmurad@yahoo.com

University Heart Journal 2017; 13(1): 36-37

\section{Introduction:}

Venous malformations are abnormally developed blood vessels with varying degrees of communication with normal veins. They are sometimes described abnormal 'vascular lakes' containing very slow moving blood. Supporting these vascular lakes is a solid component known as matrix. The ratio of spaces and matrix within a vascular malformation varies considerably from patient to patient. It can differ to some extent within different malformations within the same person. They are classified as low flow lesions meaning that they contain venous blood, which is very slow moving. Occasionally the blood moves so slowly that the blood can clot within the malformation. This usually leads to swelling and pain within the malformation.

They can occur anywhere in the body and are present at birth, although they may not become apparent until later in life. Other situations when they may become apparent are following episodes of local trauma, at puberty or pregnancy due to hormonal changes occurring at these times. Depending on their location venous malformations may cause pain, swelling, restriction of movement or cosmetic issues. Occasionally venous malformations can bleed especially if they are in a very superficial position. Treatment may be indicated because of the appearance or for associated functional problems.

Vascular malformations result from abnormal-sized vascular structures or an abnormal number of vascular structures. Vascular anomalies are a diffuse collection of vascular abnormalities that are present at birth or soon after. Historically the terminology has been complicated, descriptive, difficult to understand and a barrier to communication between specialties. The International Society for the Study of Vascular Anomalies (ISSVA) was founded after 16 years of biennial meetings with the primary goal of improving the understanding of these abnormalities. ISSVA adopted the biological classification system developed by Mulliken \& Glowacki published in
1982. ${ }^{1}$ This classification differentiated vascular anomalies due to their endothelial cell characteristics and clinical behaviour. It simply distinguished two main types of vascular anomalies: vascular tumours (grow by cellular mainly endothelial hyperplasia) and vascular malformations (localised defects in vascular morphogenesis caused by dysfunction in embryogenesis and vasculogenesis). All AVMs are present at birth, but they are not always clinically evident. Although the pathogenetic mechanisms of AVMs are not completely understood, the hemodynamic alterations that lead to the clinical manifestations of AVMs have been described well. An abnormal communication causes shunting of blood from the high-pressure arterial side to the low-pressure venous side. This creates an abnormal low-resistance circuit that steals from the highresistance normal capillary bed.

Transcatheter embolization of vascular malformations became an extremely valuable option in the treatment of these frequently complex and deeply seeded anomalies. This modality can be effectively applied alone, prior to, or in combination with surgical resection when the vascularity of the malformation must be reduced.

Most AVMs are not amenable to complete surgical excision. A lesion must be well localized for a chance at complete resection. Resectability depends on the degree of extension into adjacent structures.

A variety of percutaneous sclerotic and transarterial embolizing agents have been advocated in numerous combinations, depending on location, severity and the extent of the malformations. Absolute ethanol, bleomycin, 3\% sodium tetradecyl sulfate (STS), polidocanol, ethanolamine oleate, n-butyl cyanoacrylate, polyvinyl alcohol foam and various types of coils and polymer microspheres have all been used. ${ }^{2}$

\section{Case Report:}

A 20 year old married women admitted on July 2016 in the department of vascular surgery, Bangabandhu Sheikh 
Mujib Medical University (BSMMU), Dhaka, Bangladesh with the background history of a small bluish swelling in the left labia majora from early childhood. The diffuse swelling gradually extended into the left upper thigh, left labia majora and perineum.

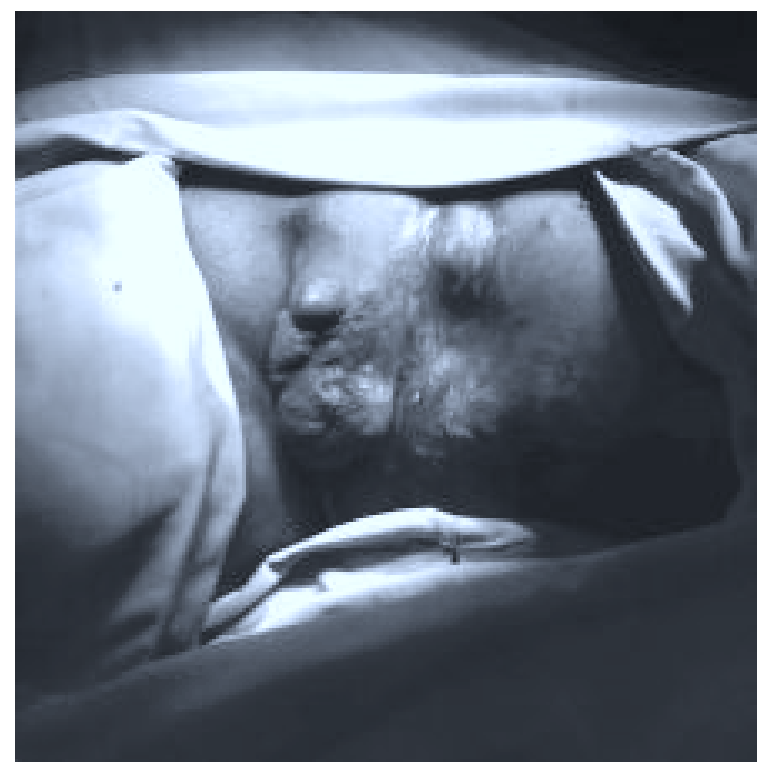

\section{Duplex Ultrasonography}

Ultrasonography with color Doppler revealed highly vascular lesion with no arterio-venous fistulous connection and diagnosed as a case of venous malformation involving left upper thigh, left labia majora and perineum.

\section{Magnetic Resonance Imaging:}

Revealed extensive lesion involving left upper thigh, left labia majora and perineum.

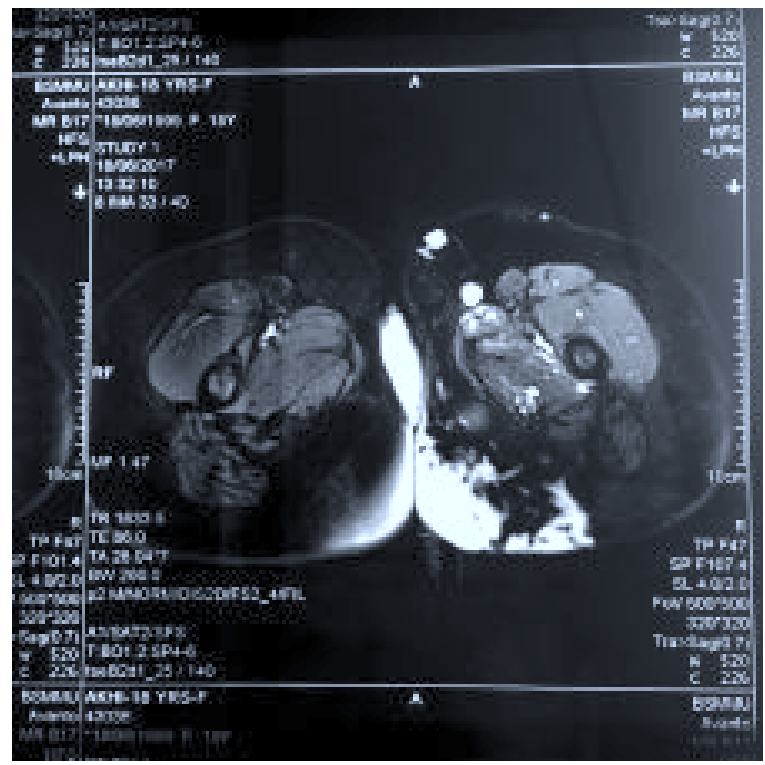

\section{Diagnosis:}

Diffuse venous malformation involving left upper thigh, left labia majora and perineum

\section{Treatment:}

Subtotal excision with adjuvant sclerotherapy is decided for the case.
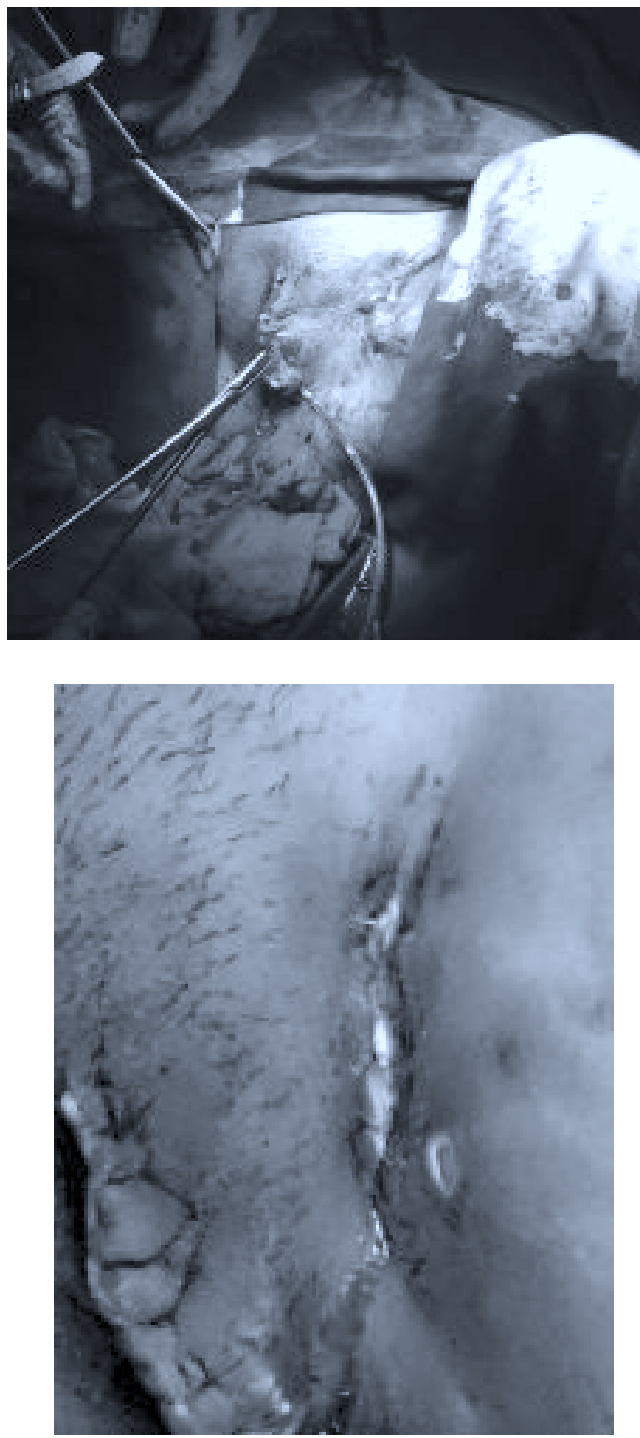

Partial excision of lesion was done. Patient is under continued sclerotherapy by sodium tetradecyl sulfate for residual lesions.

\section{References:}

1. ISSVA classification of vascular anomalies. International Society for the Study of Vascular Anomalies. Available at https:// issva.clubexpress.com/docs.ashx?id=178348. April 2014; Accessed: August 26, 2016.

2. Madani H, Farrant J, Chhaya N, et al. Peripheral limb vascular malformations: an update of appropriate imaging and treatment options of a challenging condition. The British Journal of Radiology. 2015;88(1047):20140406. doi:10.1259/bjr.20140406. 